\title{
THE EMISSION SPECTRUM OF BISMUTH MONOFLUORIDE BiF-'A' SYSTEM
}

\author{
By S. Sankaranarayanan, P. S. Narayanan and M. M. Patel* \\ (From the Department of Physics, Indian Institute of Science, Bangalore-12) \\ Received February 27, 1964 \\ (Communicated by Prof. R. S. Krishnan, F.A.sc.)
}

\section{INTRODUCTION}

THE A system of bismuth monofluoride in the visible was analysed by Howell, H. G. (1936) in emission in high frequency discharge and in the same year Morgan, F. (1936) observed this system in absorption, which showed that the lower state was the gound state of $\mathrm{BiF}$. The weak ultra-violet triplet system which was observed by Rochester, G. D. (1937) in low dispersion has subsequently been shown by Prasada Rao, T. A. and Tiruvenganna Rao, P. $(1962 a)$ to be in reality three separate systems $c_{1}, c_{2}$ and $c_{3}$. Joshi, K. C. (1961) and Rochester, G.D. (1961) made an attempt to resolve the ambiguities that existed in regard to our knowledge of the electronic levels and the transitions involved in the observed systems of $\mathrm{SbF}$ and $\mathrm{BiF}$ molecules. But Prasada Rao, T. A. and Rao, P. T. $(1962 b)$ have since shown by a rotational analysis of the five bands $(1,0),(0,0),(0,1),(0,2)$ and $(0,3)$ of the A system that the transition involved here is Hund's case $(c) \mathrm{O}^{+}\left({ }^{3} \Sigma^{-}\right)-\mathrm{O}^{+}\left({ }^{3} \Sigma^{-}\right)$. These authors have reported earlier (1962 $a$ ) that the A system. does not extend beyond $\lambda 5100$ in conformity with the observation of Howell (loc. cit). In the course of our investigations on the band spectrum of BiF, it was however noticed that similar to what has been found in recent times in $\mathrm{BiCl}$ and $\mathrm{BiBr}$, the A system of BiF does, in fact, extend beyond $\lambda 5100$ and this was possibly not noticed by them on account of the different methods of excitation employed. In addition to giving the new results and a vibrational analysis, a discussion of the dissociation energies and dissociation products of the states involved is presented here.

\section{EXPERIMENTAL}

Initial attempts to record the spectrum of $\mathrm{BiF}$ using conventional forms of the discharge tube proved a failure. Later, the following modifications

\footnotetext{
* Present Address: Reader in Physics, Faculty of Science, M. S. University, Baroda.
} 
were made. A silica tube of about $25 \mathrm{~cm}$. in length and about $1.1 \mathrm{~cm}$. in diameter was taken and a side tube was attached to it at the centre. Two hollow cylindrical nickel electrodes were slipped into the tube symmetrically disposed with respect to the centre and with a gap of about $10 \mathrm{~cm}$. in between. Pyrex adopters were attached to the ends of the silica tube with leads to the electrodes sealed in them for external connection to a transformer. One of the adopters had a side tube through which the discharge tube could be evacuated by the usual method. A glass window was cemented to the end of the side tube. Pure bismuth trifluoride $\left(\mathrm{BiF}_{3}\right)$ was taken in a separate small silica tube, one end of which was closed and the other drawn out into a narrow bore. This sample tube was placed between the electrodes. The discharge was maintained by a transformer capable of supplying $5000 \mathrm{~V}$ and $100 \mathrm{~mA}$. When the substance was strongly heated, the discharge was of bright blue colour. This discharge was photographed transversely through the side tube. The spectrum was recorded with a Hilger $\mathrm{E}_{1}$ glass spectrograph with a dispersion of about $9.6 \mathrm{~A}$ per $\mathrm{mm}$. at $\lambda 5000$, using Ilford H.P.S. plate and an exposure of about 3 hours. The band heads were measured against iron arc standard lines and the values reported here are accurate to $\pm 2 \mathrm{~cm}^{-1}$, after taking into account the nature of the band head, graininess of the plate, possible error in setting the crosswire during measurement, etc. The use of the substance in a small silica tube with a narrow opening served to maintain uniformly the same amount of vapour for a regular discharge between the electrodes and enabled us to overcome the difficulty arising from the high melting point of the substance. This was also found to prolong the life of the discharge tube which in the conventional form was attacked by fluorine, thereby impairing the vacuum. The observation of the spectrum broadside on served also to eliminate any possible trace of the nitrogen spectrum.

\section{ANALYSIS}

The recorded spectrum is reproduced in Figs. 1 and 2 of Plate XVII and the bands were found to extend up to $\lambda$ 5316. Members of the already known $(0,4)$ and $(0,5)$ sequences were developed. From considerations of intensity and the separation of the members, some of the new bands recorded were arranged with these sequences. The $(0,5)$ sequence extended up to $(14,19)$ band. The remaining bands were found to fit in as further members of the $(0,6)$ sequence. Morgan found that the 28 band heads observed by him in absorption could be accounted for by a two constant formula for the vibrational energy in the upper and lower states; but the algebraic sum of the residuals is quite large, indicating thereby that the formula is approximate. Independently, Howell derived a formula for the band heads by the 
TABLE I

$B i F$ bands of the ' $A$ ' system

\begin{tabular}{|c|c|c|c|c|}
\hline$\nu_{\text {Authors }}$ & $\nu_{\text {Morgan }}$ & $\nu_{\text {Howell }}$ & $v^{\prime} v^{\prime \prime}$ & $\nu_{\text {cal. }}$ \\
\hline .. & $24003 \cdot 8$ & $24007 \cdot 0$ & 3,0 & $24005 \cdot 5$ \\
\hline .. & $23854 \cdot 7$ & $23859 \cdot 2$ & 4,1 & $23859 \cdot 7$ \\
\hline$\cdots$ & $706 \cdot 9$ & $714 \cdot 5$ & 5,2 & $714: 9$ \\
\hline . & $641 \cdot 2$ & $640 \cdot 2$ & 2,0 & $639 \cdot 8$ \\
\hline . & $497 \cdot 2$ & $498 \cdot 3$ & 3,1 & $497 \cdot 9$ \\
\hline .. & $351 \cdot 2$ & $359 \cdot 6$ & 4,2 & $356 \cdot 5$ \\
\hline . & $271 \cdot 2$ & $271 \cdot 8$ & 1,0 & $269 \cdot 5$ \\
\hline$\cdots$ & $132 \cdot 9$ & $133 \cdot 8$ & 2,1 & $132 \cdot 1$ \\
\hline$\cdots$ & $22992 \cdot 6$ & 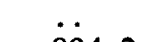 & 3,2 & $22994 \cdot 6$ \\
\hline . & $894 \cdot 2$ & $894 \cdot 2$ & 0,0 & $894 \cdot 2$ \\
\hline$\cdots$ & $630 \cdot 6$ & $631 \cdot 6$ & 2,2 & $628 \cdot 9$ \\
\hline .. & $494 \cdot 4$ & $497 \cdot 1$ & 3,3 & $495 \cdot 8$ \\
\hline . & $386 \cdot 5$ & $387 \cdot 4$ & 0,1 & $386 \cdot 6$ \\
\hline - & $360 \cdot 2$ & $\ddot{r}$ & 4,4 & $363 \cdot 1$ \\
\hline$\cdots$ & $260 \cdot 3$ & $260 \cdot 6$ & 1,2 & $258 \cdot 7$ \\
\hline$\cdots$ & $134 \cdot 6$ & $130 \cdot 0$ & 2,3 & $130 \cdot 0$ \\
\hline . & $21883 \cdot 4$ & $21884 \cdot 5$ & 0,2 & $21883 \cdot 4$ \\
\hline$\cdots$ & $761 \cdot 8$ & $762 \cdot 6$ & 1,3 & $759 \cdot 8$ \\
\hline .. & $637 \cdot 4$ & $637 \cdot 8$ & 2,4 & $635 \cdot 6$ \\
\hline .. & & $618 \cdot 6$ & 6,7 & $620 \cdot 3$ \\
\hline . & $512 \cdot 3$ & $512 \cdot 2$ & 3,5 & $21511 \cdot 2$ \\
\hline . & $385 \cdot 1$ & $385 \cdot 5$ & 0,3 & $384 \cdot 5$ \\
\hline .. & $268 \cdot 2$ & $268 \cdot 2$ & 1,4 & $265 \cdot 3$ \\
\hline .. & $148 \cdot 5$ & $147 \cdot 7$ & 2,5 & $145 \cdot s$ \\
\hline .. & $027 \cdot 0$ & $027 \cdot 7$ & 3,6 & $025 \cdot 5$ \\
\hline .. & $20906 \cdot 3$ & $20906 \cdot 1$ & 4,7 & $20906 \cdot 0$ \\
\hline .. & . & $891 \cdot 2$ & 0,4 & $890 \cdot 0$ \\
\hline . & & $785 \cdot 8$ & 5,8 & $787 \cdot 5$ \\
\hline$\cdots$ & $780 \cdot 3$ & $778 \cdot 7$ & 1,5 & $775 \cdot 2$ \\
\hline . & $\therefore$ & $668 \cdot 8$ & 6,9 & $670 \cdot 8$ \\
\hline$\ddot{0}$ & $664 \cdot 6$ & $663 \cdot 9$ & 2,6 & $659 \cdot 7$ \\
\hline 20545 & $548 \cdot 0$ & $546 \cdot 8$ & 3,7 & $544 \cdot 1$ \\
\hline 428 & $\cdots$ & $429 \cdot 6$ & 4,8 & $429 \cdot 0$ \\
\hline$\therefore$ & . & $401 \cdot 0$ & 0,5 & $399 \cdot 9$ \\
\hline 20313 & $\cdots$ & $315 \cdot 4$ & 5,9 & 314.9 \\
\hline 20291 & . & & 1,6 & $20289 \cdot 5$ \\
\hline 200 & . & $20200 \cdot 9$ & 6,10 & $202 \cdot 6$ \\
\hline 182 & . & $182 \cdot 0$ & 2,7 & $178 \cdot 4$ \\
\hline 090 & . & $090 \cdot 0$ & 7,11 & $092 \cdot 5$ \\
\hline 070 & . & $068 \cdot 7$ & 3,8 & $067 \cdot 2$ \\
\hline 19985 & . & $19981 \cdot 0$ & 8,12 & $19985 \cdot 3$ \\
\hline 958 & . & 957.8 & 4,9 & $956 \cdot 4$ \\
\hline 883 & . & $\cdots$ & 9,13 & $881 \cdot 5$ \\
\hline 869 & . & $\ldots$ & 14,17 & $869 \cdot 2$ \\
\hline
\end{tabular}


The Emission Spectrum of Bismuth Monefluoride BiF-'A' System

Table I (Contd.)

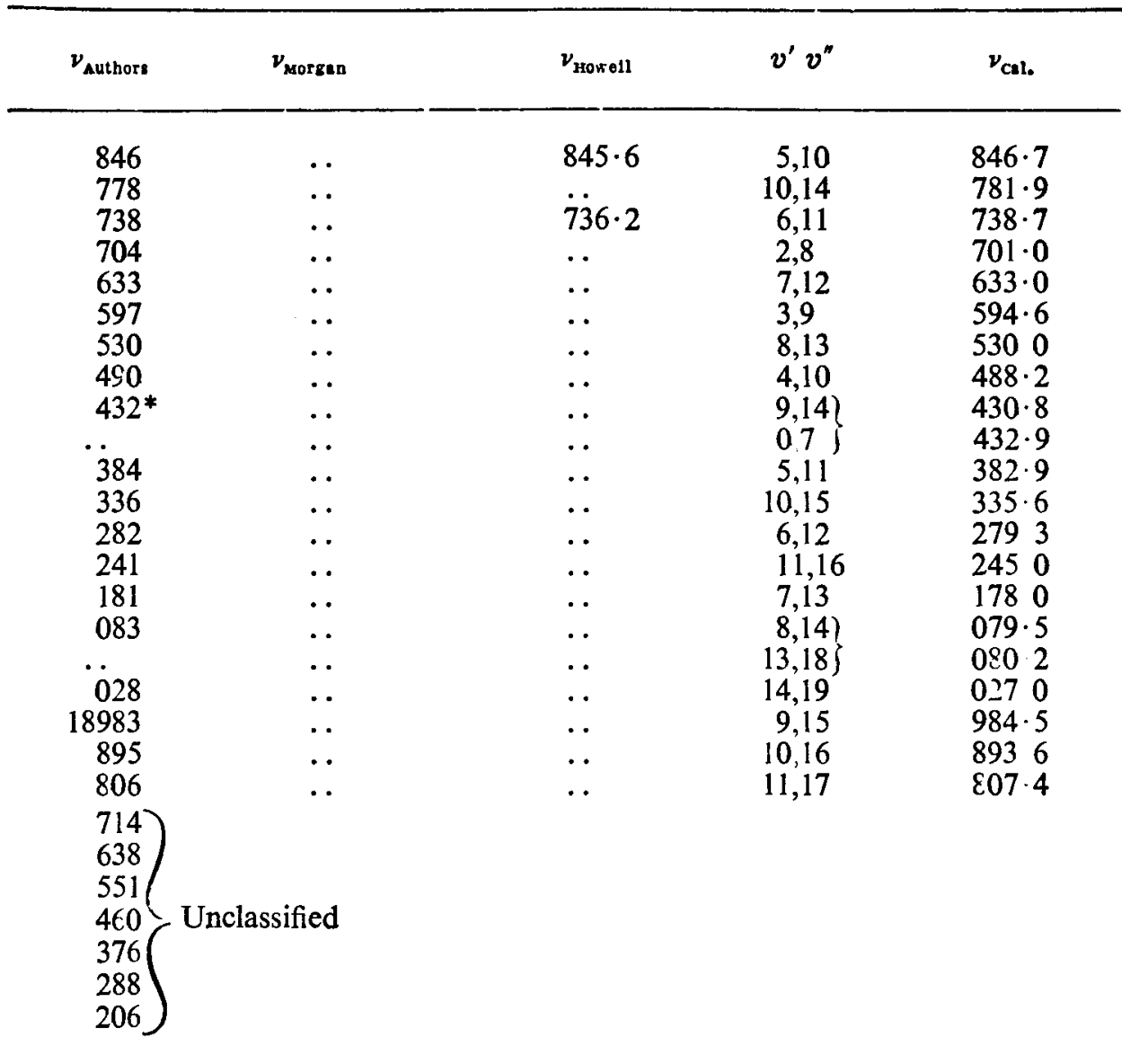

* Masked by $\lambda 5144.48$ of $\mathrm{Bi}$.

method of least squares and he found it necessary to add a cubic term to the upper state. The residuals, in this case ranged from -2.8 to 2.3 and their algebraic sum was $-0.4 \mathrm{~cm} .^{-1}$, which is small enough to justify the formula. When these two formulae were employed it was found that the differences between observed values and those calculated with Morgan's formula were very large. Howell's formula yielded values for band heads which were closer to observed values but the differences were still appieciable. Though it is tetter to derive the formula using band origin data, in this instance Prasada Rao, T. A. and Rao, P. T. (loc. cit.) have carried out the rotational analysis only on five bands and it was possible to calculate the first two terms for the ground state only. Consequently, in Howell's band head equation 
the constants for the upper state were retained as such and the ground state constants refined to yield the following equation:

$$
\begin{aligned}
\nu= & 22959 \cdot 9+381 \cdot 0\left(v^{\prime}+\frac{1}{2}\right)-3 \cdot 0\left(v^{\prime}+\frac{1}{2}\right)^{2} \\
& +0.1\left(v^{\prime}+\frac{1}{2}\right)^{3}-512 \cdot 0\left(v^{\prime \prime}+\frac{1}{2}\right)+2 \cdot 19\left(v^{\prime \prime}+\frac{1}{2}\right)^{2} .
\end{aligned}
$$

The lower state constants $\omega_{e}{ }^{\prime \prime}=512$ and $\omega_{e}{ }^{\prime \prime} x_{e}{ }^{\prime \prime}=2 \cdot 19$ compare very well with the values $\omega_{e}{ }^{\prime \prime}=\sim 512.6$ and $\omega_{e}{ }^{\prime \prime} x_{e}{ }^{\prime \prime}=\sim 2 \cdot 3$ that alone can be derived from the band origin data of Prasada Rao, T. A. and Rao, P. T. (loc. cit.). The wave-numbers of the band heads, their vibrational assignment and the calculated value for the same are given in Table I. As in the present study with the $E_{1}$ spectrograph, the spectrum was not recorded on the high frequency side $\nu$ Morgan and $\nu$ Howell have also been included in the table for comparison with $\nu$ calc. Seven other faint bands in the region $\lambda$ 5316-5492 were also observed. These bands, however, could not be classified into this system, though they resemble very much the bands belonging to the A system. The usual precautions were taken to ensure that the carrier of the spectrum was really the BiF molecule (see Howell, loc. cit.).

\section{Discussion}

The ground state $\mathrm{X}-\mathrm{O}^{+}\left({ }^{3} \Sigma^{-}\right)$can be derived from the normal atoms $\mathrm{Bi}\left({ }^{4} \mathrm{~S}_{3 / 2}\right)$ and $\mathrm{F}\left({ }^{2} \mathrm{P}_{3 / 2}\right)$ and therefore it can be assumed that in the bismuth monofluoride molecule the ground state dissociation products are the normal atoms. A reasonable value of the dissociation energy $\left(\mathrm{D}_{e}{ }^{\prime \prime}\right)$ may be obtained from the relation (Varshni, Y. P., 1957)

$$
\mathrm{D}_{e}=\frac{5 k_{e} r_{e}^{2} \mathrm{~W}}{2\left(\omega_{e} x_{e} \mu_{\mathrm{A}} r_{e}^{2}-9 \mathrm{~W}\right)}
$$

where $\mathrm{W}=2 \cdot 1078 \times 10^{-16}$ and $k_{e}$ is the force constant obtained from the relation

$$
k_{e}=5 \cdot 8883 \times 10^{-2} \mu_{A} \omega_{e}^{2}
$$

where $\omega_{e}$ is the frequency, $\mu_{\mathrm{A}}$ the reduced mass, $r_{e}$ and $\omega_{e} x_{e}$ are the equilibrium internuclear distance and the anharmonicity constant obtained experimentally. For the ground state of BiF these are $2 \cdot 045 \AA$ and $2.19 \mathrm{~cm}^{-1}$ respectively. This dissociation energy $\mathrm{D}_{e}$ " comes out to be $15662 \mathrm{~cm}^{-1}$ The heat of dissociation $\mathrm{D}_{0}{ }^{\circ}$ on the other hand may be obtained from thermochemical data using the relation

$$
D(B i-F)=\Delta H_{f}^{0}(B i)+\Delta H_{f}^{0}(F)-\triangle H_{f}^{0}(B i-F)
$$


where $\triangle \mathrm{H}_{f}{ }^{0}(\mathrm{Bi}), \triangle \mathrm{H}_{\mathrm{f}}{ }^{0}(\mathrm{~F})$ and $\triangle \mathrm{H}_{f}{ }^{0}(\mathrm{Bi}-\mathrm{F})$, the standard heats of formation, are known from experimental measurements to be $49 \cdot 7,18 \cdot 3$ and $7.0 \mathrm{~K}$. cal./mol. respectively (Rossini et al., 1952). Although a correction has to be applied to this to obtain $\triangle \mathrm{H}_{0}{ }^{0}$ which alone corresponds to the dissociation energy $\mathrm{D}_{0}{ }^{0}$ (Herzberg, G., 1950), it can be seen that the correction is very small in this case (in the absence of $\mathrm{C}_{p}$ data an approximate correction can be made classically) and the bond strength from thermochemical data is found to be $\mathrm{D}_{0}{ }^{0}=\sim 21335 \mathrm{~cm} .^{-1}$ or $2.65 \mathrm{ev}$. Hence it appears that the true dissociation energy is between $15600 \mathrm{~cm}^{-1}$ and $21300 \mathrm{~cm} .^{-1}$ and it is in all probability around $20,000 \mathrm{~cm}^{-1}(2 \cdot 48 \mathrm{ev}$.). Using Varshni's relation, the dissociation energy for the upper state $\left(D_{e}{ }^{\prime}\right)$ comes out to be $7850 \mathrm{~cm}^{-1}$ or $0.97 \mathrm{ev}$. This low value is in agreement with the Hund's case $(c)$ coupling found to exist in the molecule for A system by Prasada Rao, T. A. and Rao, P. T. (loc. cit.). From the relation $\mathrm{E}_{\mathrm{M}}+\mathrm{D}^{\prime}=\mathrm{E}_{\mathrm{A}}+\mathrm{D}^{\prime \prime}$ where $\mathrm{E}_{\mathrm{M}}$ is the electronic excitation energy of the upper state, $D^{\prime}$ the dissociation energy of the excited state, $E_{A}$ the atomic excitation energy and $D^{\prime \prime}$ the dissociation energy of the lower state, $E_{A}$ is found to be about $10800 \mathrm{~cm}^{-1}$ Hence the dissociation products of the upper state $A$ are not the normal atoms but with one of the atoms in the excited state. The first excited level of fluorine is $102412 \mathrm{~cm} \cdot .^{-1}$ above the ground state and may therefore be left out of consideration. On the other hand, the first excited level of $\mathrm{Bi}$ atom $\left({ }^{2} \mathrm{D}_{3 / 2}\right)$ lies $11418 \mathrm{~cm} .^{-1}$ above the ground state ${ }^{4} S_{3 / 2}$ (Bacher, R. F. and Goudsmit, S., 1932) and this value is sufficiently close to the estimated value of $E_{A}$ to conclude that the dissociation products in the upper state are $\mathrm{Bi}\left({ }^{2} \mathrm{D}_{3 / 2}\right)$ and $\mathrm{F}\left({ }^{2} \mathrm{P}_{3 / 2}\right)$ atoms. That this conclusion is correct is shown by the fact that bismuth and fluorine atoms in these states respectively, by correlation rules, can give rise to another $\mathrm{O}^{+}\left({ }^{3} \Sigma^{-}\right)$molecular state which corresponds to the upper state identified by Prasada Rao, T. A. and Rao, P. T. (loc. cit.).

\section{ACKNOWLEDGEMENT}

Our sincere thanks are due to Prof. R. S. Krishnan for his kind encouragement and interest in our work.

\section{SUMMARY}

The observation of (A-X) system of BiF has been extended up to $\lambda 5316$ and twenty new bands belonging to this system have been recorded. The band heads could be represented by the following equation:

$$
\begin{aligned}
\nu= & 22959 \cdot 9+381 \cdot 0\left(v^{\prime}+\frac{1}{2}\right)-3 \cdot 0\left(v^{\prime}+\frac{1}{2}\right)^{2} \\
& +0 \cdot 1\left(v^{\prime}+\frac{1}{2}\right)^{3}-512 \cdot 0\left(v^{\prime \prime}+\frac{1}{2}\right)+2 \cdot 19\left(v^{\prime \prime}+\frac{1}{2}\right)^{2},
\end{aligned}
$$


Seven other faint bands in the region $\lambda$ 5316-5492 have also been reported, which, however, could not be classified. By our analysis of the present data and from known thermochemical data it has been deduced that the ground state dissociation energy is, in all probability, around $20000 \mathrm{~cm} . .^{-1}(\sim 2.5 \mathrm{ev}$.) and that the dissociation products are the normal $\mathrm{Bi}$ and $\mathrm{F}$ atoms. The dissociation energy of the upper state and the correlation rules have been used to show that the dissociation products in the upper state are very likely to be $\mathrm{Bi}$ atom in the excited state ${ }^{2} \mathrm{D}_{3 / 2}$ and $\mathrm{F}$ atom in its ground state $\left({ }^{2} \mathrm{P}_{3 / 2}\right)$.

\section{REFERENCES}

Bacher, R. F. and Goudsmit, S. Atomic Energy States, McGraw-Hill Book Co., N.Y., 1932. Herzberg, G.

.. Spectra of Diatomic Molecules, Van Nostrand, 1950.

Howell, H. G.

.. Proc. Roy. Soc. Lond., 1936, 155, 141.

Joshi, K. C.

.. Proc. Phys. Soc., 1961, 78, 610.

Morgan, F.

.. Phys. Rev., 1936, 49, 41.

Prasada Rao, T. A. and Tiruvenganna Rao, $\mathbf{P}$. Ind. Journ. Phys., $1962 a, 36,85$.

.. Can. Journ. Phys., $1962 b, 40,1077$.

Rochester, G. D.

.. Phys. Rev., 1937, 51, 486.

.. Proc. Phys. Soc., 1961, 78, 614.

Rossini, F. D., Wagman, D. D., "Selected values of chemical thermodynamic properties," Evans, W. H., Levine, S. and Jaffe, I. Circular of the National Bureau of Standards, Washington, D.C., 1952.

Varshni, Y. P.

.. Rev. Mod. Phys., 1957, 29, 664. 

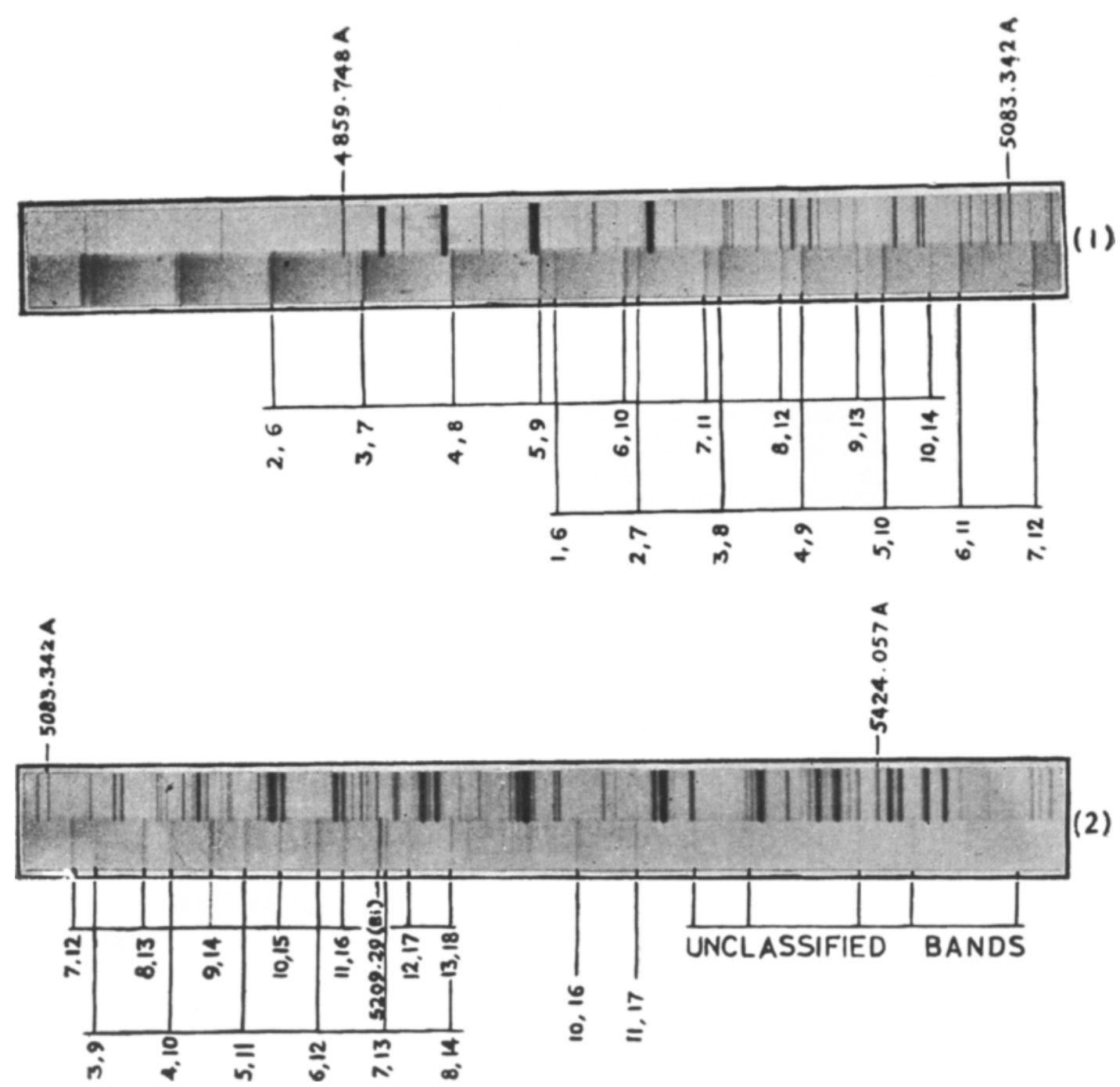

Fig. 1. 'A' system from $4785 \AA$ to $5092 \AA$.

FIG. 2. Extension of 'A' system. (BiF Bands) 